\title{
La notion de conceptualisation en didactique des langues
}

Jean-François Bourdet et Raphaële Fouillet

\section{OpenEdition}

Édition électronique

URL : https://journals.openedition.org/rdlc/2669

DOI : $10.4000 /$ rdlc.2669

ISSN : 1958-5772

Éditeur

ACEDLE

Référence électronique

Jean-François Bourdet et Raphaële Fouillet, « La notion de conceptualisation en didactique des langues ", Recherches en didactique des langues et des cultures [En ligne], 15-1 | 2018, mis en ligne le 02 janvier 2018, consulté le 21 septembre 2021. URL : http://journals.openedition.org/rdlc/2669; DOI : https://doi.org/10.4000/rdlc.2669

Ce document a été généré automatiquement le 21 septembre 2021.

\section{c) (i)}

Recherches en didactique des langues et des cultures is licensed under a Creative Commons AttributionNonCommercial-NoDerivatives 4.0 International License 


\title{
La notion de conceptualisation en didactique des langues
}

\author{
Jean-François Bourdet et Raphaële Fouillet
}

« Nous voulons enseigner à parler et à écrire

français et non enseigner le contenu des

grammaires. » (Besse, 1974 : 39)

\section{Introduction}

1 La grammaire, qu'on choisisse de l'ignorer, de la minimiser ou de la placer au cœur de l'enseignement des langues, n'en constitue pas moins un aspect incontournable et souvent problématique. On est ainsi passé de la grammaire comme principal accès à la langue étrangère à la grammaire implicite des méthodes audio-orale et structuro-gobale audio-visuelle (SGAV) en quelques dizaines d'années, sans pour autant trouver une approche qui ferait consensus. Avec l'entrée dans l'ère communicative, la tendance est à la " conceptualisation grammaticale ", pour les tenants d'un enseignement explicite de la grammaire. Parce que le terme est profondément inscrit dans le champ du Français langue étrangère (FLE) mais aussi parce qu'il semble détourné des intentions de départ de son concepteur, il nous a paru important de le questionner.

Introduite par Henri Besse dans les années 1970, l'idée de conceptualisation a séduit, circulé, fait l'objet de malentendus et subi des transformations. Qu'entendait alors exactement Henri Besse par conceptualisation grammaticale? Qu'en est-il de la notion de départ au regard de l'évolution vers la méthode actionnelle ou encore de l'introduction de l'émergentisme et de l'énaction dans le champ de la didactique des langues? Quelles critiques peut-on formuler de cette approche? Comment les concepteurs de manuels de FLE s'en sont-ils emparés depuis les premiers ouvrages parus sous l'étiquette « approche communicative » et par conséquent, comment "font-ils faire» de la grammaire aux enseignants et aux apprenants? Les quelques éléments de réponse apportés à ces interrogations aboutissent au moins à une certitude: la grammaire est un pan de l'enseignement du FLE qui mérite encore réflexion. 


\section{La notion de conceptualisation en FLE : une idée originale d'Henri Besse}

3 H. Besse introduit la notion de conceptualisation dans un article paru en 1974, dans le bulletin de liaison et d'information trimestriel Voix et images du CREDIF, sous le titre Les exercices de conceptualisation ou la réflexion grammaticale au niveau 2. Les exercices de conceptualisation ont été proposés pour la première fois dans le manuel Interlignes.

\section{Le contexte de création des « exercices de conceptualisation »}

4 L'idée de conceptualisation apparait dans la nomination d'un type d'exercice grammatical qu'H. Besse met au point dans le contexte SGAV de l'enseignement des langues des années 1970. On peut avancer que son élaboration s'est construite sur une insatisfaction relative à la méthode SGAV en général, et à son approche grammaticale en particulier, insatisfaction qui l'a mené à une interrogation et une réflexion sur la grammaire en tant que notion, sur son rôle dans l'apprentissage des langues étrangères et sur une pédagogie adaptée à son appropriation par les élèves.

$5 \quad \mathrm{H}$. Besse ${ }^{1}$ formule une critique générale vis-à-vis de la méthode SGAV en avançant qu'aborder une langue étrangère par l'oral (dialogues enregistrés) et le visuel (images projetées sur un écran) ne convient pas à tous les élèves. Il cite ainsi l'exemple de Japonais inscrits dans une culture éducative essentiellement scripturale, désorientés par l'approche acoustico-visuelle de la langue française. Il se reconnait pleinement dans la perspective fonctionnelle de G. Gougenheim et du Français fondamental, tout en rejetant les exercices structuraux qui, uniquement axés sur l'idée de faire acquérir les structures syntaxiques par leur répétition et leur manipulation, évacuent le sens des phrases. Ajoutons que l'époque est aux reproches formulés de plus en plus ouvertement à l'encontre de la méthode SGAV, mais aussi à la réflexion menée sur l'enseignement des langues étrangères à un niveau 2 (la méthode SGAV ayant étant pensée pour les débutants). Une interrogation plus large semble cependant dominer la conception des exercices de conceptualisation: qu'entend-on par "grammaire»? H. Besse répond à cette question dans l'ouvrage devenu une référence en didactique des langues, Grammaires et didactique des langues (1984), soit dix ans après l'article présentant les exercices de conceptualisation. Les sept ou huit acceptions du terme circulant à l'époque sont réduites aux trois suivantes : 1) un certain fonctionnement interne caractéristique d'une langue donnée; 2) l'explicitation plus ou moins méthodique de ce fonctionnement; 3) la méthode d'explicitation suivie (Besse \& Porquier, $1991: 11$ ).

6 L'objectif didactique visé par H. Besse à travers les exercices de conceptualisation n'est pas de réintroduire l'enseignement grammatical explicite déductif - dans lequel l'élève apprend une règle façonnée par des grammairiens puis l'applique - mais de viser l'appropriation par l'élève de la connaissance intériorisée de la langue qui lui est étrangère au départ. Pour $\mathrm{H}$. Besse, cette appropriation passe par un retour réflexif personnel sur les structures que l'élève emploie parce qu'il les a incorporées. Il s'agit là d'une prise de position, d'une conviction pourrait-on dire, forgée au cours de ses années d'expériences en tant qu'examinateur envoyé dans de nombreuses classes de français à travers le monde pour le compte du CREDIF. 
7 Les « exercices de conceptualisation » renouent avec la réflexion grammaticale opposée à l'enseignement implicite de la grammaire et avec l'utilisation d'un métalangage, qu'il soit traditionnel ou inventé par les élèves. Cette approche semble jouer un rôle charnière avec l'époque communicative qui se profile. Cette dernière, en effet, ne s'adresse plus à l'individu que l'on élève mais à celui qui apprend et qui est le principal acteur de son apprentissage. Voici peut-être une des raisons pour lesquelles l'idée de conceptualisation a fait florès dans la didactique du FLE.

\section{La conceptualisation grammaticale d'après Henri Besse}

8 Le terme conceptualisation appartient au champ philosophique, sa première attestation remontant à 1936. Besse (1974: 38$)$ s'explique sur le choix de ce mot et sur le sens qu'il lui confère de la manière suivante :

C'est l'idée qu'on se fait d'un certain fonctionnement de la phrase, idée qui peut s'appliquer à nombre d'autres phrases quand aucune contrainte ne vient limiter ce fonctionnement.

9 Car ce qui est en jeu, ce n'est pas l'apprentissage de la grammaire, mais bien la réflexion grammaticale. Il s'agit de passer d'une assimilation inconsciente des structures de la langue à un recul conscient sur le fonctionnement de cette dernière. $\mathrm{H}$. Besse, tout en souhaitant rompre avec une certaine approche de la grammaire, inscrit sa proposition dans un esprit de continuité de la méthode SGAV où « au niveau 2, l'élève connaît par la pratique beaucoup de constructions et leur fonctionnement de manière implicite » (Besse, 1974 : 38). On peut supposer que le mot « exercice » a été choisi, entre autres, afin que les «exercices de conceptualisation» représentent une solution de remplacement aux «exercices structuraux». H. Besse souligne que ceux-ci ne constituent pas une technique d'enseignement de la grammaire mais un outil permettant de structurer l'apprentissage de l'élève, de l'accompagner dans son intériorisation de la grammaire.

H. Besse considère en effet en 1974 que l'élève doit avoir assimilé au préalable un certain nombre de structures syntaxiques et de vocabulaire, grâce à son exposition assez longue à la langue étrangère (niveau 2), pour pouvoir pratiquer les exercices de conceptualisation. La conceptualisation devient en quelque sorte un levier permettant de faire remonter à la surface ce qui est inconscient chez l'élève: des structures grammaticales et du vocabulaire qu'il a faits siens à force de répéter et de mémoriser des dialogues. On pourrait avancer que le rôle de l'enseignant consiste à faire prendre conscience à l'élève de ce qu'il ne sait pas qu'il sait déjà. La remontée du fonctionnement de la langue à la surface de la conscience s'effectue par le biais de la mise en mots par l'élève lui-même. «Peu importe qu'elle [l'explication] recouvre ou non les catégories de grammairiens" (Besse, 1974 : 38), car il s'agit d'amener l'élève à élaborer une règle de fonctionnement qui peut être provisoire et évoluer au fil de son apprentissage de la langue - lui permettant de vérifier la possibilité de produire un énoncé donné. Ces constantes de fonctionnement mises au jour par l'élève s'appliquent plus à des « microsystèmes » qu'à «l'ensemble du système de la langue » (Besse, 1974 : 38).

11 Le but des exercices de conceptualisation étant d'amener les élèves à verbaliser, conscientiser, valider, invalider, modifier les règles de fonctionnement qu'ils ont inconsciemment élaborées pour pouvoir produire des énoncés acceptables par les locuteurs natifs, le matériau de base des exercices de conceptualisation est constitué de leurs productions mêmes. Celles-ci reflétant un état de la grammaire qu'ils sont en train 
de construire. La phrase est l'unité de base à partir de laquelle l'observation est menée. La paraphrase est la clé de voûte de la mise en mots des règles de fonctionnement des microsystèmes, car elle permet un accès aux nuances. La démarche d'H. Besse consiste non pas à orienter immédiatement le regard des élèves vers la structure syntaxique mais à l'orienter d'une part vers le sens véhiculé par tel ou tel énoncé, et d'autre part vers la structure qui permet de l'exprimer. A partir du moment où le sens est dégagé, et par conséquent les nuances qui existent entre tel et tel énoncés, on cherche à faire correspondre ces nuances à une structure précise. Les exercices de conceptualisation remplissent le double objectif de "permettre d'affiner le vocabulaire des élèves tout en développant leur syntaxe » (Besse, $1974: 42$ ).

Les étapes concrètes mais nullement figées des exercices de conceptualisation sont les suivantes :

13 1) recueillir des productions des élèves ;

2) si possible, les écrire au tableau, ce qui facilitera la réflexion;

3) faire classer et analyser les productions ;

4) faire mettre en mots ce qui a été compris du fonctionnement de la langue ;

5) faire réemployer la structure dégagée ;

6) compléter ou modifier la règle élaborée par les élèves lorsqu'une production est erronée.

Les exercices de conceptualisation peuvent être menés à partir d'énoncés porteurs de sens très proches mais aussi à partir d'énoncés présentant des structures très proches. Un dernier aspect des exercices de conceptualisation est qu'ils se font en groupe. Cela permet d'homogénéiser les connaissances des élèves de la classe qui peuvent ainsi se corriger et d'instaurer un climat d'entraide entre les élèves puisque ceux qui ont une intuition plus grande du fonctionnement de la langue aident les autres (Besse, $1974: 39$ ).

\section{La notion de conceptualisation dans le champ de la didactique}

La notion de conceptualisation correspond à une aspiration de changement commune aux acteurs de l'enseignement, qui se manifeste sous la forme d'une nouvelle approche de l'enseignement des langues. Des recherches aux manuels, tout le champ de la didactique est bouleversé par l'approche communicative.

\section{Une notion dans l'air du temps}

La notion de conceptualisation reprise par Besse et Porquier dans leur ouvrage de 1984 joue un rôle essentiel dans le développement d'une approche grammaticale de type inductif. Elle trouve écho dans des réflexions conduites dans les années 80 sur les catégories d'apprentissage et l'interlangue. Pour la première notion, on citera la thèse de Dalgalian, Lieutaud et Weiss (1982) ; pour la seconde, les propositions de Selinker (1972) introduisant le concept d'«interlangue » ont joué un rôle central. Les didacticiens vont donc trouver, à travers ces deux notions, un socle épistémologique permettant de construire des référents méthodologiques privilégiant l'analyse de corpus et la construction bottom up de règles de fonctionnement de la langue en cours d'apprentissage. Cette perspective d'autonomisation du traitement des données 
langagières est étayée par le développement d'une nouvelle approche pédagogique qui se désigne comme "communicative" et fait appel à une participation renouvelée des étudiants, rebaptisés «apprenants " pour marquer le rôle central qu'ils ont à jouer dans le traitement de l'input des contenus des cours donnant lieu à un intake qui met en jeu un travail d'élaboration personnel très proche des phases de conceptualisation identifiées par Besse et Porquier.

17 Cette nouvelle approche tire également parti des évolutions de la linguistique qui, de manière concomitante, se diversifie en linguistique pragmatique et linguistique de l'énonciation, allant au-delà des propositions de variations établies par la sociolinguistique des années 60 .

18 C'est donc un faisceau de phénomènes/référents convergents qui se trouvent synthétisés dans l'ouvrage de Besse et Porquier en 1984. À bien des égards, ceux-ci tirent leçon de données déjà existantes plus qu'ils ne les annoncent. Mais le grand intérêt de leur ouvrage, et l'impact durable qui va en découler, est de présenter sous une forme claire et scientifiquement bien établie, une lecture de phénomènes disséminés et souvent flous dans l'espace des pratiques enseignantes. Il entraine aussi, comme conséquence notable, la prééminence reconnue (à tort ou à raison) aux phases de conceptualisation dans une séquence pédagogique; phases par rapport auxquelles les étapes - antérieures - de sensibilisation et d'identification, - ultérieures - de systématisation et de transfert apparaissent un peu comme des faire-valoir.

\section{La notion de conceptualisation dans les manuels}

On peut remarquer que dès la fin des années 70, les premiers manuels FLE d'inspiration communicative proposent sous forme d'activités de repérage des corpus langagiers susceptibles de traitement métalinguistique par inférence de régularités repérées par les apprenants, ensuite mises en forme de manière plus normative afin d'aboutir à des règles de fonctionnement. Considérons quelques exemples pris dans des manuels anciens et récents: Archipel 1 (1982), Campus 2 (2002), Edito 2 (2006), Latitudes 3 (2010), Alter Ego+ B2 (2015).

\section{Archipel 1}

On trouve dans Archipel 1 (Courtillon, 1982) des activités ayant recours à l'induction, plus que de véritables séquences de conceptualisation. On est effectivement à la limite de l'exercice (répétitivité, convergence, prévisibilité) et de l'activité (repérage, interprétation). C'est toute l'ambiguïté des perspectives formelles, telles qu'elles sont envisagées dans une approche communicative: on préfère renvoyer à l'usage par réemploi en situation qu'à un travail effectif de réflexivité, voire de métacognition, sur les valeurs d'usage des catégories grammaticales. C'est par exemple au sein des pages intitulées Un peu de grammaire qu'on rencontre de telles activités; ainsi page 107, sous le bandeau Remarquez, apparaît une liste d'énoncés porteurs d'expressions de quantité :

Il y a beaucoup de monde, il y a plein de monde.

Il y a assez de chocolat, ça suffit.

Il y a trop de pain, mais pas assez de jambon.

Il y a un peu de sauce, vous en voulez un peu? 
21 (facilités par l'emploi des italiques dans le texte original), ne rend guère possible une véritable conceptualisation des formes qui renverrait à des règles d'usage en contexte. Le recours obligé au corpus pré constitué dans les manuels permet surtout, nous semble-t-il, de travailler la forme (comme dans un paradigme) et de se livrer à des manipulations syntaxiques fort proches d'exercices de systématisation (en poursuivant la liste).

final, Archipel 1 révèle un état des lieux intéressant, pris dans la tension entre participation apprenante, y compris dans la réflexion grammaticale souhaitée, et réalisation éditoriale sous forme de manuel devant structurer ces apprentissages. La solution médiane adoptée est celle d'un recours à l'induction, cadrée dans des activités de repérage qui sont de fait assez éloignées de ce que $\mathrm{H}$. Besse entend par exercice de conceptualisation. Cette tension va se retrouver dans le matériel pédagogique mis à disposition dans les décennies suivantes.

\title{
Campus 2
}

Campus 2 (Girardet \& Pêcheur, 2002) présente des sections où la réflexion sur la langue recourt à l'induction. L'introduction du livre de l'élève ne comporte pas d'informations sur l'approche grammaticale, le terme même de « grammaire » n'apparaissant pas. Il faut se reporter au livre du professeur pour trouver des pistes permettant d'identifier le type d'approche pratiquée. On trouve dans l'introduction du Livre du professeur l'information suivante: Chaque leçon de Campus 2 se présente comme une suite d'activités motivantes qui cherchent à optimiser l'apprentissage. Il est intéressant de comparer deux sections de cette introduction dont la juxtaposition nous semble poser question. À propos de la progression, les auteurs indiquent que le recyclage des acquisitions est permanent. L'automatisation des constructions grammaticales, par exemple, se poursuit tout au long de la méthode. Ce qui semble conduire à une grammaire plutôt implicite. Mais, à la page suivante, on trouve l'exemplification suivante :

\begin{abstract}
Pour travailler les verbes qui expriment les sentiments et qui déterminent souvent l'emploi du subjonctif, on propose deux lettres que s'adressent deux amies d'enfance qui vivent l'une en France et l'autre en Australie (leçon 5(4), p. 68). La découverte des lettres se fait grâce à la technique du dévoilement progressif. A chaque phrase, les étudiants notent ce qu'ils apprennent sur les deux correspondantes et formulent des hypothèses. La compréhension du texte et le repérage des formes qui expriment les sentiments sont donc portés par un projet : rassembler le plus possible d'informations sur les deux femmes.
\end{abstract}

24 Voilà qui laisse songeur : de quelle teneur est ce dévoilement ? Et, s'il concerne l'emploi des formes, comment se met en place la construction d'une règle d'usage ? La page 68 demande effectivement de noter des informations concernant les épistolières, mais, quant aux formes, il s'agit d'un simple exercice de systématisation.

Dans une autre activité page 34, on demande aux apprenants de «noter les arguments pour ou contre » (activité 2), puis d' « observer les formes de l'expression de la volonté et de l'obligation » (activité 3 ) ; cette consigne étant assortie de deux exemples : « Ils veulent supprimer » et «Qu'est-ce que tu veux qu'ils fassent? ».

Deux remarques peuvent être faites. D'abord quant à la formulation de la consigne qui rappelle le manuel Archipel 1 (Observez). Ce type de formulation s'apparente un peu à 
l'invocation d'un supposé pouvoir d'induction, mais qu'en sera-t-il en situation de classe : observez risque de se révéler peu productif ; relevez et classez serait déjà plus incitatif parce qu'outillant la démarche d'une orientation (Classez, donc réfléchissez à des modes de catégorisation) et, si on le souhaite, de critères de classement (Classez les formes en vous servant du sens des verbes introducteurs; Notez ce qui les rapproche ou les différencie). Le renvoi à la forme est si prégnant qu'il semble difficile d'y insérer l'écart nécessaire à la réflexivité.

\section{Edito B2}

Edito B2 est un manuel publié en 2006 par le même éditeur que celui de Campus 2 et dû à Heu et Mabilat. L'avant-propos du livre de l'élève indique qu'un accent particulier est mis sur l'approfondissement de la grammaire et du vocabulaire. L'apprenant est actif : il passe du stade de la découverte, à la déduction et au réemploi.

Voyons ce que cela donne dans les faits. La page 24, par exemple, présente une activité susceptible de donner lieu à conceptualisation sur l'emploi de l'indicatif et du subjonctif après un verbe. À vrai dire, il s'agit d'une séquence explicitement grammaticale, mais comportant la possibilité d'une esquisse de conceptualisation du fait de la justification demandée quant à l'emploi des modes (et renvoyant à la page $14 \mathrm{du}$ manuel). Il ne s'agit donc pas d'une réelle conceptualisation sur corpus puisque les modes sont déjà connus et travaillés, mais d'un support de conceptualisation potentielle si on utilise la page pour demander une théorisation du jeu modal; ce qui est possible à partir de la justification d'emploi contenue dans la consigne. C'est, dans ce manuel, la page qui se rapproche le plus d'une séquence de conceptualisation.

\section{Latitudes 3}

L'avant-propos du livre de l'élève revendique une approche actionnelle et ne met pas l'accent sur l'apprentissage grammatical. Si la démarche s'appuie sur l'observation et la réflexion, la grammaire n'est en effet évoquée qu'au gré de la rubrique S'entraîner contenant des exercices de grammaire. On peut donc s'interroger sur le lien établi entre observation et exercices.

C'est la page 27 de Latitudes 3 (Loiseau et al., 2010), qui offre matière à réflexion. Sous l'intitulé Comment choisir la place des adjectifs ?, on demande aux apprenants d'observer des phrases et de discuter de la place des adjectifs, puis de lire un texte et de répondre à des questions guidant une réflexion grammaticale. Indéniablement, une possibilité de réflexion langagière existe ici, point de départ d'une construction de règle d'emploi liée à la place de l'adjectif.

\section{Alter Ego B2}

31 Nous prendrons, pour terminer cette brève recension, un dernier exemple dans l'un des manuels les plus récents : Alter Ego B2 (Antier et al., 2015). L'avant-propos revendique :

l'implication de l'apprenant dans son apprentissage, ce dernier étant supposé actif, il développe ses aptitudes d'observation et de réflexion, autant de stratégies qui l'amènent progressivement vers l'autonomie. 
32 grammaire, les doubles pages Des mots et des formes sont là pour « assurer la grammaire, avec exemples et tableaux récapitulatifs ». Il convient donc d'examiner comment la grammaire est ainsi assurée. La page 24 traite de l'emploi du passé composé versus imparfait. Elle propose diverses activités, sous l'intitulé S'exercer et donne pour consigne de compléter une biographie en conjuguant les verbes à l'imparfait ou au passé composé puis de justifier l'emploi/le choix (?) de ces temps. Comme on le voit, rien là que de très ordinaire: il s'agit en fait d'un exercice d'application à visée systématisante. Le point intéressant est proposé par la seconde partie de la consigne qui renvoie à la possibilité de justifier l'emploi/le choix (?) des temps. On fait donc appel à une réflexivité langagière reposant sur une théorisation. Celle-ci peut être acquise ou non, et ce dernier cas permettrait de développer une phase de conceptualisation en reprenant le texte pour le transformer en corpus d'identification des formes et de construction de règles justifiant leur emploi. matériel éditorial disponible depuis 30 ans. Comme on l'a vu, il s'agit essentiellement d'activités de repérage (plutôt formel) ou de confirmation (plutôt sémantique) sur les catégories grammaticales. Nous n'avons trouvé aucune séquence correspondant réellement à ce que serait une phase de conceptualisation remontant du repérage formel à une interprétation sémantique de l'occurrence des phénomènes identifiés, mais bien plutôt des moments de classe à orientation réflexive, faisant appel à l'induction. Une explication pourrait être formulée à partir de ce que nous disions ci-dessus à propos d' Archipel 1. La participation des apprenants à l'ensemble des phases d'apprentissage est aujourd'hui constituante d'une doxa largement partagée, d'autant que les approches communicative et actionnelle, le CECRL, l'ont nourrie et répandue. Il faut donc lui faire place ; mais, d'un autre côté, il est difficile de contraindre et d'intégrer dans une linéarité de type progressif, la multiplicité des interlangues à l'œuvre dans les phases de conceptualisation et qui n'est rien moins que linéaire au vu de la complexité et de la diversité des facteurs impliqués. Pris dans cette dichotomie, les auteurs de manuels et leur direction éditoriale trouvent une solution médiane dans le recours à l'induction, mais sans aller au-delà.

Au moins n'est-il pas interdit aux enseignants de se servir de ces activités pour mettre en place des activités plus proches de la conceptualisation telle qu'envisagée par $\mathrm{H}$. Besse. Ce que ce dernier redoutait s'est donc produit dans la mesure où, 25 ans après la création des exercices de conceptualisation, il constate, notamment en s'appuyant sur l'avant-propos des auteurs de C'est le Printemps 1 (1976), que les exercices de conceptualisation "ont souvent été interprétés comme un simple retour à la technique inductive traditionnelle ». (Besse, 2000).

\section{Regard critique sur les exercices de conceptualisation}

Les exercices de conceptualisation tels qu'ils ont été conçus ont en quelque sorte échappé à leur concepteur qui n'hésite pas à leur reconnaître certaines limites et certaines difficultés. La démarche mérite également d'être examinée à l'aune de notions émergentes en didactique des langues. 


\section{Une auto-critique des exercices de conceptualisation} élèves ayant été scolarisés et ayant reçu un enseignement grammatical dans leur langue de départ. Par ailleurs, plus les élèves connaissent la langue étrangère, moins les aspects syntaxiques et sémantiques abordés apparaissent dans les ouvrages de grammaire, la crainte de ne pas être capable d'accompagner les élèves dans leur mise en mots du fonctionnement d'un microsystème pouvant alors freiner les professeurs dans leur élan pour adopter une telle approche de la grammaire. On compte parmi les autres critiques formulées: une préparation importante de la part du professeur; une concentration exigeante pour les élèves, qui, conjuguée à l'ennui que les exercices de conceptualisation génèrent chez certains, conduit à limiter le recours aux exercices de conceptualisation autant du point de vue de leur fréquence que de leur durée (environ 40 minutes).

Besse (2000) constate cependant que ce qui était destiné au départ à des élèves de niveau 2 a été adapté à des niveaux moins avancés. Ce qui rejoint une réflexion formulée dans un article de 1976, qui l'amène à revenir sur le présupposé du niveau des élèves pour mettre en place des exercices de conceptualisation:

Ces démarches [basées sur la conceptualisation] n'étaient présentées comme applicables qu'à un niveau assez avancé, alors qu'elles le sont beaucoup plus tôt dans l'apprentissage, si on use de procédures qui permettent aux étudiants d'acquérir plus rapidement, dans leurs pratiques de classe, le sentiment de ce qui est possible et ne l'est pas dans la langue étrangère, et si on admet l'usage «métalinguistique » d'une langue de départ. (Besse, $1976: 258$ )

1 reconnaît également que la démarche pédagogique de conceptualisation est résolument inductive (Besse, 1974: 39), mais il s'attache à montrer qu'elle ne se confond en rien avec la grammaire inductive. Il précise en effet que les exercices de conceptualisation ont bien été conçus dans le prolongement de la méthode SGAV mais dans la quasi ignorance de la place de l'induction dans l'histoire de l'enseignement des langues (Besse, 2000). Il met ainsi en évidence différents points de divergence entre les deux démarches. La grammaire inductive en effet :

1. part d'exemples soigneusement sélectionnés par l'enseignant;

2. peut partir des productions des élèves mais pour aboutir à la formulation précise d'une loi de la langue. En ce sens, H. Besse écrit qu'elle est un enseignement vivant de la grammaire

3. veut s'appliquer à l'échelle de l'ensemble de la langue et aboutir à une règle générale ; 4. s'appuie sur une observation tangible des structures.

\section{Une critique au regard l'émergentisme et l'« énaction »}

40 Un point peut mettre en question la prééminence des phases de conceptualisation : celui d'une conception émergentiste ${ }^{2}$ des apprentissages (Miras \& Narcy-Combes, 2014) qui minore la possible conduite enseignante du phasage de la dynamique apprenante au profit d'une écoute plus attentive des évolutions des acteurs envisagées sous l'angle de l' « énaction ${ }^{3}$ ", à la suite de Varela (1991).

41 Il y a là matière à débat, mais il nous paraît utile de signaler ainsi ce qui peut apparaitre comme une limite du champ d'application de la notion de conceptualisation. La relecture 
du cadre épistémologique de la didactique des langues, qui se dessine à travers les notions d'énaction et d'émergentisme, a pour mérite de replacer la conceptualisation dans un cadre plus large et d'aider à comprendre son rayonnement depuis trente ans. L'intuition supposée de la langue chez les élèves par la démarche de conceptualisation y trouve en effet un prolongement et un recadrage à la lumière d'une vision plus globale de l'individu apprenant.

La conceptualisation, telle qu'elle a été comprise et utilisée par les acteurs du FLE, a rendu possible un équilibre entre guidage enseignant (ce qui peut être interprété a minima comme le socle même du métier) et autonomisation apprenante, apparaissant ainsi comme un lieu d'échange et de renforcement mutuel de ces deux perspectives. La confrontation métalinguistique qu'elle autorise conjoint régularité repérée (côté apprenant) et règle de référence attestable (côté enseignant), toutes deux liées dans un jeu d'échange.

Elle a offert à la fois un cadre de référence (notion didactique dominante), un outil de structuration méthodologique (phase centrale de la classe, construction de l'apprentissage) et une technique pédagogique opératoire (exposition à un corpus constitué, recours aux processus d'inférence, exposition des régularités identifiés). C'est cette homothétie unifiante de l'espace pédagogique dans son ensemble qui explique aussi le succès d'une démarche réflexive de l'enseignement/apprentissage de la grammaire.

\section{Conclusion}

La riche expérience d'Henri Besse, en tant qu'enseignant de français langue étrangère et examinateur envoyé par le CREDIF dans de nombreuses classes de français à travers le monde, a nourri sa réflexion sur l'enseignement et l'apprentissage de la grammaire et l'a conduit à concevoir les " exercices de conceptualisation » ou " réflexion grammaticale ", une approche qui tranchait avec la méthode SGAV alors dominante dans les années 70. Le mot "conceptualisation ", a, dès lors, occupé une place majeure dans l'enseignement/ apprentissage du FLE. Une analyse de manuels publiés au cours des trente dernières années montre cependant que sous l'étiquette de "conceptualisation", les manuels proposent en réalité une grammaire inductive explicite. Cette assimilation pourrait trouver une explication dans un aspect de la démarche des exercices de conceptualisation. Ceux-ci, en effet, s'appuient entièrement sur l'intuition grammaticale de l'élève que l'on sollicite pour élaborer activement une grammaire commune au groupe. Celui qui est devenu l'apprenant avec l'approche communicative est donc partie prenante de son apprentissage. Ce serait alors plutôt l'idée de participation active de l'apprenant qui a été retenue comme représentative de la proposition d'Henri Besse. Reste que l'impact de la notion de conceptualisation, même si elle a été déformée par rapport à l'idée initiale, est indéniable. L'enseignement des langues perdrait beaucoup à se priver d'une réflexion dans laquelle peut se développer une autonomisation du rapport au matériau linguistique. C'est sans doute là que se découvre une base réelle pour une autonomisation si souvent recherchée et si rarement développée. 


\section{BIBLIOGRAPHIE}

Besse, H. (1974). « Les exercices de conceptualisation ou la réflexion grammaticale au niveau 2 ». Voix et Images du CREDIF 2. pp. 39-44.

Besse, H. (1976). « Propositions pour une didactique de la grammaire ». In Revue de Phonétique Appliquée, $\mathrm{n}^{\circ}$ 39-40. pp. 231-262.

Besse, H. \& Porquier R. (1991). Grammaires et didactique des langues. [1984]. Paris : Didier.

Besse, H. (2000). Propositions pour une typologie des méthodes de langues. Tome 2, chapitre 4. pp. 680-685.

Coste, F., « Incarnation, cognition et représentation : comment les sciences cognitives pensentelles le corps ? », Tracés. Revue de Sciences humaines [En ligne], 2 | 2003, mis en ligne le 11 mai 2009.

Dalgalian, G., Lieutaud, S. \& Weiss, F. (1981). Pour un nouvel enseignement des langues. Paris : Clé International.

Miras, G. \& Narcy-Combes, J.-P. (2014). « Conséquences sur les pratiques d'une prise en compte intégrée des théories socioconstructiviste et émergentiste ». Travaux et documents. Mars, $\mathrm{n}^{\circ} 46$. pp. 15-26.

Robert, J.-P. (2008). Dictionnaire pratique de didactique du FLE. Paris: Ophrys.

Selinker, L. (1972). « nterlanguage ». International Review of Applied Linguistics, n 10. pp. 219-231.

Varela, F., Thompson, E. \& Rosch, E. (1991). The Embodied Mind: Cognitive Science and Human Experience. MIT Press (trad. en français par Havelange, V. (1996). L'Inscription corporelle de l'esprit : sciences cognitives et expérience humaine. Paris : Seuil).

\section{Corpus}

Antier, M. \& al. (2015). Alter Ego + B2. Paris : Hachette.

Courtillon, J. (1982). Archipel 1. Paris : Didier.

Girardet, J. \& Pêcheur, J. (2002). Campus 2. Paris : Didier.

Heu, E. \& Mabilat, J.-J. (2006). Edito B2. Paris : Didier.

Loiseau, Y. \& al. (2010). Latitudes 3. Paris : Didier.

\section{NOTES}

1. Séminaire du 20 mai 2011 organisé par le DILTEC/GRAC à l'Université Paris 3 intitulé : Pour une relecture, quarante ans plus tard, des « exercices de conceptualisation ».

2. On peut la caractériser comme l'intégration de la pensée complexe aux situations d'apprentissage. Elle se qualifie par: la non-linéarité; l'absence de pré-structuration; une polarité multiple non-structurée. 
3. Modèle créé par Varela en 1991 selon lequel la cognition est «l'avènement conjoint d'un monde et d'un esprit à partir de l'histoire des diverses actions qu'accomplit un être dans le monde » (Coste, 2003).

\section{RÉSUMÉS}

Le terme conceptualisation, introduit par Henri Besse dans les années 1970, s'est durablement inscrit dans le champ de la didactique des langues à la faveur de l'approche communicative. Ce qu'il recouvre ne correspond cependant pas tout à fait à l'idée originelle dont on a principalement retenu l'aspect inductif, comme le montre une analyse de plusieurs manuels de FLE édités sur une période de 30 années. On relève par ailleurs que son ancrage dans le contexte particulier des années 1970 ne permet pas à la conceptualisation grammaticale de répondre de manière pleinement satisfaisante au cadre de l'approche actionnelle ni de coïncider avec les notions d'émergentisme et d'énaction apparues très récemment dans la didactique des langues.

In the communicative approach to foreign language learning, the term "conceptualization ", introduced by Henri Besse in the 1970s has become a durable element in the field. However, its coverage today does not correspond entirely to the original idea of which we mainly retain the inductive aspect, as shown in an analysis of several textbooks of FLE over a period of 30 years. Furthermore, being rooted in the particular context of the 1970s, it does not allow grammatical conceptualization to deal adequately with the so-called «task-based approach» nor does it correspond to the notions of emergentism and enactivism which have appeared very recently in the didactics of languages.

\section{INDEX}

Mots-clés : conceptualisation, didactique des langues, grammaire

Keywords : conceptualization, didactics of languages, grammar

\section{AUTEURS}

\section{JEAN-FRANÇOIS BOURDET}

CREN - Le Mans (EA 2661) - Le Mans Université

Après avoir enseigné le Français comme langue étrangère, avoir été formateur d'enseignants et responsable pédagogique de l'Alliance française de Paris, Jean-François Bourdet a rejoint Le Mans Université où il a développé divers enseignements de master puis a activement collaboré à la création de l'offre de formation en ligne de l'université. Spécialiste des dispositifs de formation à distance, il a mené de nombreuses missions de formation et d'expertise des centres de langues en France et à l'étranger. Il développe sa recherche dans le cadre du CREN-Le Mans, particulièrement sur les ingénieries de formation instrumentées par le numérique (conception, expertises, interfaces dédiées au tutorat). Il est Professeur des Universités, Responsable de la recherche à l'UFR Lettre, Langues et Sciences Humaines, et Directeur adjoint de l'École doctorale 
ELICC (Éducation, Langages, Cognition, Cliniques).

Courriel : jean-francois.bourdet[at]univ-lemans.fr

\section{RAPHAËLE FOUILLET}

CREN - Le Mans (EA 2661) - Le Mans Université

DILTEC/GRAC (EA 2288) - Université Sorbonne Nouvelle - Paris 3

Raphaële Fouillet est docteure en Didactique des langues et des cultures de l'Université Sorbonne Nouvelle. Sa thèse et les travaux s'y rapportant portent principalement sur la didactique de la grammaire du français en milieu italophone à travers l'étude de manuels. Rattachée au CREN-Le Mans, ses recherches portent également sur l'enseignement des langues à l'école primaire. Elle enseigne actuellement le Français langue étrangère dans une association et collabore

régulièrement à la création de contenus grammaticaux dans des manuels de Français langue étrangère pour les Editions Maison des Langues.

Courriel : r.fouillet11[at]gmail.com 